\title{
Nuclear reaction path and inertial mass in the self-consistent collective coordinate method
}

Kai Wen, Fang Ni, and Takashi Nakatsukasa*

Center for Computational Sciences and Faculty of Pure and Applied Sciences, University of

Tsukuba, Tsukuba 305-8577, Japan

\begin{abstract}
The adiabatic self-consistent collective coordinate (ASCC) method and its application to nuclear structure and reaction are presented. The decoupled one-dimensional collective paths are determined for pairing modes in $\mathrm{Sn}$ isotopes and for low-energy fusion reaction of ${ }^{16} \mathrm{O}+{ }^{16} \mathrm{O}$. We show that the collective path is often significantly different from our intuitive choice, such as the pair gap and the relative coordinate between two nuclei.
\end{abstract}

The 26th International Nuclear Physics Conference

11-16 September, 2016

Adelaide, Australia

${ }^{*}$ Speaker. 


\section{Introduction}

Development of microscopic theories for large amplitude collective motion is a long-standing problem in physics of nuclear structure and reaction [四]. It is natural to assume that the collective motion is described by time evolution of a mean-field state (Slater determinant). This leads to a semi-classical description of the collective motion. However, in experiments, nuclear collective motion is usually identified by large transition probabilities and characteristic properties of energy eigenstates. Thus, the classical dynamics of the mean-field state should be requantized.

In order to achieve the quantization, it is desirable to introduce a small number of canonical collective variables which describe the collective motion of the mean-field state. In many cases, these collective variables are assumed to be mean values of certain one-body operators intuitively chosen. The choice is trivial for some cases, such as the center-of-mass motion of a nucleus. In general, however, it is not at all easy to choose them. One of the main subjects of the present paper is the microscopic determination of such one-body operators. More precisely speaking, we self-consistently determine particle-hole and hole-particle parts of the operator, that is nothing but generators of the collective variables [四]. These generators define the tangent space at every point (mean-field state) in the collective subspace.

Another issue in the nuclear collective motion is the inertial mass. It is known that the simplest generator-coordinate method (GCM) does not give a good description of the dynamics, and its Gaussian overlap approximation (GOA) cannot produce a proper inertial mass [3]. A similar problem is also present in the cranking formula [3] which has been most commonly used in many applications [ [⿴囗大 , []. The problem is associated with improper treatment of the time-odd mean-field effects. In contrast, the random-phase approximation (RPA), defined as the small-amplitude limit of the time-dependent mean-field theory, properly takes into account the time-odd effects and is able to reproduce the exact total mass for the center-of-mass motion. We provide an extension of the RPA equation for non-equilibrium states, which is used for determination of the generators and the inertial masses.

In section 几, we derive basic equations of our method. They are presented here in a different form from references [ [ [], The formulation is based on the classical Hamilton's equation, which is given in reference $[$ 圆] and similar to the one in reference $[$ [ $]$. We derive equations to determine tangent vectors of the collective subspace, which are identical to the particle-hole (hole-particle) components of the generators. In section [3, the method is applied to studies of nuclear pairing dynamics and nuclear fusion reaction. The reaction path and inertial mass are self-consistently determined. Finally, section 1 summarize the conclusion.

\section{Adiabatic self-consistent collective coordinate method}

We present derivation of the basic equations to determine a decoupled collective subspace (path) and inertial mass. An extension of the present formalism can be found in reference [ 6$]$.

\subsection{Classical Hamiltonian}

The time-dependent mean-field equation determines the time evolution of a Slater determinant $|\phi(t)\rangle$. Using the Thouless' theorem and a suitable mapping of coefficients [ [ $]$ ], it can be written in 
terms of classical canonical variables $\left\{\xi^{\alpha}(t), \pi_{\alpha}(t)\right\}$ as $|\phi(\xi, \pi)\rangle$ where $\alpha=1, \cdots, M$. The number of variables $M$ corresponds to the number of independent particle-hole (two-quasiparticle) degrees of freedom. The time-dependent mean-field equation can be written as the Hamilton's equation of motion; $\dot{\xi}^{\alpha}=\partial H / \partial \pi_{\alpha}$ and $\dot{\pi}_{\alpha}=-\partial H / \partial \xi^{\alpha}$. Here the classical Hamiltonian is introduced as the expectation value of the original Hamiltonian $\hat{H}$

$$
H(\xi, \pi) \equiv\langle\phi(t)|\hat{H}| \phi(t)\rangle=\langle\phi(\xi, \pi)|\hat{H}| \phi(\xi, \pi)\rangle
$$

Assuming that the collective motion is slow, the Hamiltonian is expanded up to the second order in momenta $\pi_{\alpha}$;

$$
H(\xi, \pi)=V(\xi)+\frac{1}{2} B^{\alpha \beta}(\xi) \pi_{\alpha} \pi_{\beta},
$$

where the reciprocal inertial mass tensor is given by $B^{\alpha \beta}(\xi) \equiv \partial^{2} H /\left.\partial \pi_{\alpha} \partial \pi_{\beta}\right|_{\pi=0}$, and the Einstein summation convention is adopted for upper and lower indexes.

\subsection{Decoupled collective motion by point transformation}

We now try to find a motion described by collective variables $\left(q^{1}, p_{1}\right)$, well decoupled from the rest of the degrees of freedom, $\left\{q^{a}, p_{a}\right\}_{a=2, \cdots, M}$. Here, we assume that the collective coordinate is one-dimensional (1D). The point transformation, $q^{\mu}(\xi)$ with $\mu=1, \cdots, M$, leads to the Hamiltonian $\bar{H}$ as

$$
\bar{H}(q, p)=\bar{V}(q)+\frac{1}{2} \bar{B}^{\mu v}(q) p_{\mu} p_{v}
$$

where

$$
\bar{V}(q)=V(\xi(q)), \quad \text { and } \quad \bar{B}^{\mu v}(q)=q_{, \alpha}^{\mu} B^{\alpha \beta} q_{, \beta}^{v} .
$$

Here, the derivative is denoted by a comma such as $q_{, \alpha}^{\mu} \equiv \partial q^{\mu} / \partial \xi^{\alpha}$. If we restrict the transformation to the linear transformation, $q_{, \alpha}^{\mu}$ become constant. In general, they are position-dependent and should be determined locally at each point in the collective space.

The decoupling subspace is defined by the condition that the system in the collective subspace will stay within the subspace: $\quad q^{a}=p_{a}=0 \Rightarrow \dot{q}^{a}=\dot{p}_{a}=0 \quad(a=2, \cdots, M)$. Using the Hamilton's equation of motion, this condition can be written as the following [ [ $]$.

$$
\text { (i) } \bar{V}_{, a}=0, \quad \text { (ii) } \bar{B}^{a 1}=0, \quad \text { (iii) } \bar{B}_{, a}^{11}=0, \quad \text { for } \quad a=2, \cdots, M \text {, }
$$

in the collective subspace $\left(q^{a}=0\right.$ ). If all the conditions (i-iii) of ([D.J) are satisfied, the decoupling is exact. In nuclear physics, the exact decoupling occurs only for the trivial center-of-mass motion. We are interested in low-energy collective motion, $\left(q^{1}, p_{1}\right)$, which is approximately decoupled from other intrinsic degrees of freedom, $\left\{q^{a}, p_{a}\right\}_{a=2, \cdots, M}$. In the next subsection [2.3, we derive a set of equations to determine the tangent vector $q_{, \alpha}^{1}$ and its inverse $\xi_{, 1}^{\alpha}$, using the conditions of ([2.5).

\subsection{Basic equations}

The condition (i) in (2.5]) can be rewritten as

$$
V_{, \alpha}-\bar{V}_{, 1} q_{, \alpha}^{1}=0
$$


which means that the gradient of potential $V(\xi)$ is parallel to the collective path $q^{1}$. This can be regarded as the minimization of $V(\xi)$ with a constraint on $q^{1}$.

$$
\delta\left\{V(\xi)-\lambda q^{1}(\xi)\right\}=0 .
$$

We call equation (‥6) moving mean-field equation. For a given tangent vector $q_{, \alpha}^{1}$, the solution of (R.6) determines a point in the collective space, namely, a Slater determinant $|\phi(\xi, \pi=0)\rangle$.

Next, we differentiate (2.6) with respect to $q^{1}$.

$$
\left(V_{, \alpha \beta}-\bar{V}_{, 1} q_{, \alpha \beta}^{1}\right) \xi_{, 1}^{\beta}=\bar{V}_{, 11} q_{, \alpha}^{1}
$$

Because of the condition (i), the left hand side can be written in terms of the covariant derivative as

$$
V_{, \alpha \beta}-\bar{V}_{1} q_{, \alpha \beta}^{1}=V_{, \alpha \beta}-\bar{V}_{\mu} q_{, \alpha \beta}^{\mu}=V_{, \alpha \beta}-\Gamma_{\alpha \beta}^{\gamma} V_{, \gamma} \equiv V_{; \alpha \beta} \xi_{, 1}^{\beta},
$$

with the affine connection of $\Gamma_{\alpha \beta}^{\gamma}=\xi_{, \mu}^{\gamma} q_{, \alpha \beta}^{\mu}$. This means that $\left\{q^{\mu}\right\}$ is the normal (geodesic) coordinates, namely $\bar{\Gamma}_{v \rho}^{\mu}=0$. Equation (2.8) leads to

$$
B^{\alpha \gamma} V_{; \gamma \beta} \xi_{, 1}^{\beta}=B^{\alpha \gamma} \bar{V}_{, 11} q_{, \gamma}^{1}=\bar{B}^{11} \bar{V}_{, 11} \xi_{, 1}^{\alpha}
$$

where we have used

$$
B^{\alpha \beta} q_{, \beta}^{1}=\bar{B}^{11} \xi_{, 1}^{\alpha},
$$

which is equivalent to the decoupling condition (ii) in ([2.5). Using the fact that both $B^{\alpha \beta}$ and $V_{; \alpha \beta}$ are tensors of the rank 2, we can also derive equations for left eigenvectors as

$$
q_{, \alpha}^{1} B^{\alpha \gamma} V_{; \gamma \beta}=q_{, \alpha}^{1} \xi_{, \mu}^{\alpha} \bar{B}^{\mu \rho} \bar{V}_{, \rho v} q_{, \beta}^{v}=\bar{B}^{1 \rho} \bar{V}_{, \rho v} q_{, \beta}^{v}=\bar{B}^{11} \bar{V}_{, 1 v} q_{, \beta}^{v}=\bar{B}^{11} \bar{V}_{, 11} q_{, \beta}^{1}
$$

where we use the derivative of (i) with respect to $q^{1}$ at the last equation. These equations (․ㅁ) and (2.12) are harmonic equations to determine the normal-mode vectors, generalized to nonequilibrium states. At the equilibrium with $V_{, \alpha}=0$, they become the conventional harmonic approximation with the eigenfrequency $\left(\bar{B}^{11} \bar{V}_{, 11}\right)^{1 / 2}$.

The covariant derivative $V_{; \alpha \beta}$ requires the calculation of the affine connection that is given by $\Gamma_{\alpha \beta}^{\gamma}=\xi_{, 1}^{\gamma} q_{, \alpha \beta}^{1}$ on the 1D collective subspace satisfying ([2.6). To eliminate the awkward quantity $q_{, \alpha \beta}^{1}$, we differentiate the reciprocal collective inertial mass $\bar{B}^{11}$ with respect to $\xi^{\beta}$. Using the condition (iii) of ([2.5) , we obtain

$$
\bar{B}_{, 1}^{11} q_{, \beta}^{1}=B_{, \beta}^{\alpha \gamma} q_{, \alpha}^{1} q_{, \gamma}^{1}+2 B^{\alpha \gamma} q_{, \gamma}^{1} q_{, \alpha \beta}^{1} .
$$

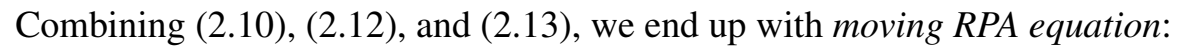

$$
\mathscr{M}_{\beta}^{\alpha} q_{, \alpha}^{1}=\omega^{2} q_{, \beta}^{1}, \quad \mathscr{M}_{\beta}^{\alpha} \xi_{, 1}^{\beta}=\omega^{2} \xi_{, 1}^{\alpha}
$$

where

$$
\mathscr{M}_{\beta}^{\alpha} \equiv B^{\alpha \gamma} V_{, \gamma \beta}+\frac{1}{2} B_{, \beta}^{\alpha \gamma} V_{, \gamma}, \quad \omega^{2} \equiv \bar{B}^{11} \bar{V}_{, 11}+\frac{1}{2} \bar{B}_{, 1}^{11} \bar{V}_{, 1} .
$$

Equations ([2.6) and (2.14) are our basic equations. These are identical to the adiabatic selfconsistent collective coordinate (ASCC) method proposed in reference [[]]. In the ASCC formalism 
[M, ㅁ] , the equations are given by a variational form in terms of the generators of the collective variables, $\hat{P}(q)$ and $\hat{Q}(q)$, locally defined at each state on the subspace. They are composed of the zeroth $\left(p^{0}\right)$, first $\left(p^{1}\right)$, and second-order $\left(p^{2}\right)$ equations in collective momentum $p$. The correspondence between the present formulation and those in reference [U, [ []] is identified by some relations, such as

$$
\delta\langle\phi(q)|\hat{Q}(q)| \phi(q)\rangle=\delta \xi^{\alpha} q_{, \alpha}^{1}, \quad \delta\langle\phi(q)|\hat{P}(q)| \phi(q)\rangle=\delta \pi_{\alpha} \xi_{, 1}^{\alpha} .
$$

Then, we can easily find out that the $p^{0}, p^{1}$, and $p^{2}$ equations of the ASCC exactly match with

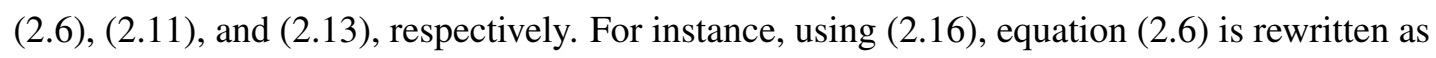

$$
\delta\langle\phi(q)|\hat{H}-\lambda \hat{Q}(q)| \phi(q)\rangle=0,
$$

that is identical to the $p^{0}$ equation of the ASCC [प, []].

\section{Applications}

In this section, we present our recent application of the ASCC method. Before presenting our results, let us comment on the scaling of the collective coordinate and quantization.

The obtained 1D collective subspace is parameterized by the coordinate $q^{1}$. Using a local scale transformation $\left(q^{1}, p_{1}\right) \rightarrow\left(\alpha q^{1}, p_{1} /\left(\alpha+\alpha^{\prime} q^{1}\right)\right)$ with a function $\alpha\left(q^{1}\right)$, the reciprocal collective inertial mass for the scaled coordinate $\alpha q^{1}$ is given by $\bar{B}^{11}\left(q^{1}\right) \times\left(\alpha+\alpha^{\prime} q^{1}\right)^{2}$. Therefore, a proper choice of the function $\alpha$ may lead to a constant inertial mass $\bar{B}^{11}$. The quantization of the collective Hamiltonian, (2.3) with $\mu=v=1$, is performed on this coordinate system, which is nothing but the Pauli's prescription.

In order to obtain an intuitive understanding of the reaction dynamics, it may be convenient to choose the new coordinate as the expectation value of a certain one-body operator $\hat{Q}$, such as the multipole moment, the relative distance between colliding nuclei, etc. This is equivalent to the scaling $\alpha=Q\left(q^{1}\right) / q^{1}$ where $Q\left(q^{1}\right) \equiv\left\langle\phi\left(q^{1}\right)|\hat{Q}| \phi\left(q^{1}\right)\right\rangle$. When the expectation value $Q\left(q^{1}\right)$ becomes roughly constant as a function of $q^{1}, Q^{\prime} \approx 0$, we have $\bar{B}^{Q Q} \approx 0$, which means a large inertial mass $M(Q)=1 / \bar{B}^{Q Q}$. If the oneto-one correspondence between $q^{1}$ and $Q\left(q^{1}\right)$ is lost, $M(Q)$ diverges. Thus, the behavior of $M(Q)$ is a kind of indicator, how suitable the choice of the one-body operator $\hat{Q}$ is.

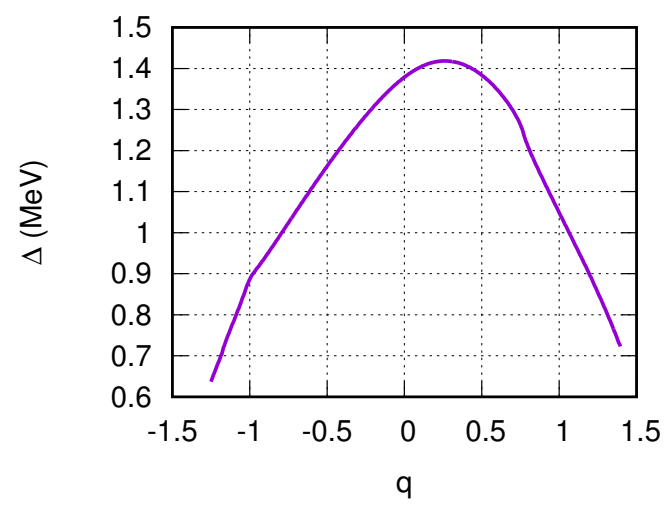

Figure 1: Neutron pair gap $\Delta$ as a function of the collective coordinate $q^{1}$ for ${ }^{112} \mathrm{Sn}$. We set $\bar{B}^{11}=1 \mathrm{MeV}$ to determine the scale of $q^{1}$ (dimensionless).

\subsection{Collective motion associated with nuclear pairing}

First, we apply the method to a nuclear structure problem. In the past, we studied collective motion associated with the shape (quadrupole) degrees of freedom [U, 目]. In this paper, we present our recent study on the collective motion associated with the pairing dynamics. Conventionally, 
the pair gap $\Delta$ has been often regarded as a collective variable. A question is how suitable this assumption is.

We adopt a simple monopole pairing model for $\mathrm{Sn}$ isotopes, $\hat{H}=\sum_{l} \varepsilon_{l}-g / 4 \hat{P}^{\dagger} \hat{P}$, where $\hat{P}$ is the monopole pairing operator. Five spherical single-particle energies for $\left\{d_{5 / 2}, g_{7 / 2}, s_{1 / 2}, d_{3 / 2}, h_{11 / 2}\right\}$ are included as $\varepsilon_{l}(l=1, \cdots, 5)$ which are obtained with a standard Woods-Saxon potential. The pairing force strength $g$ is determined so as to reproduce the experimental neutron pair gap for ${ }^{112} \mathrm{Sn}$. Using the coherent-state representation, the mean-field (BCS) dynamics can be mapped into the classical Hamiltonian (D. from the ground BCS state $\xi_{0}$, and solve the moving RPA equation (2.14) to obtain $q_{, \alpha}^{1}$ and $\xi_{, 1}^{\alpha}$. The reciprocal inertial mass is calculated as $\bar{B}^{11}=q_{, \alpha}^{1} B^{\alpha \beta} q_{, \beta}^{1}$. Then, using a small step $\delta q$, we move to the next point $\xi^{\alpha}=\xi_{0}^{\alpha}+\delta q \times \xi_{, 1}^{\alpha}$. Repeating this procedure, we obtain a series of points in the five-dimensional space $\left(\xi^{1}, \cdots, \xi^{5}\right)$, which compose the $1 \mathrm{D}$ collective path in the 5D space.

Upon the obtained collective path for ${ }^{112} \mathrm{Sn}$, change of the neutron pair gap $\Delta$ is shown in figure $\mathbb{W}$. It is not monotonic, which suggests that, adopting the energy gap $\Delta$ as a collective variable is not appropriate, since the one-to-one correspondence between $q^{1}$ and $\Delta$ is lost. In fact, the inertial mass $M(\Delta)$ would diverges at $q^{1} \approx 0.3$ where $d \Delta / d q^{1}=0$.

Requantizing the $1 \mathrm{D}$ collective Hamiltonian, we obtain the energies of excited $0^{+}$states. They are shown in Table $\mathbb{W}$. The original pairing Hamiltonian is known as the Richardson model and exactly solvable. The results show reasonable agreement with those of the collective Hamiltonian.

\begin{tabular}{l|rr}
\hline & ASCC & Exact \\
\hline${ }^{112} \mathrm{Sn}$ & 2.61 & 2.80 \\
${ }^{114} \mathrm{Sn}$ & 2.46 & 2.56 \\
${ }^{116} \mathrm{Sn}$ & 2.58 & 2.61 \\
${ }^{118} \mathrm{Sn}$ & 2.24 & 2.98 \\
${ }^{120} \mathrm{Sn}$ & 2.89 & 2.77 \\
\hline
\end{tabular}

Table 1: Calculated excitation energies of excited $0^{+}$states in units of MeV, compared with exact values.

\subsection{Subbarrier fusion reaction of ${ }^{16} \mathrm{O}+{ }^{16} \mathrm{O}$}

Fusion reaction at low energy involves a variety of shape degrees of freedom in addition to the relative motion between two nuclei. Here we aim at microscopic determination of the reaction path using the ASCC method.

We adopt the Bonche-Koonin-Negele (BKN) energy density functional [Q] and three-dimensional coordinate representation for single-particle orbitals. In order to solve the moving mean-field equation (ㄷ.6), we use the imaginary-time method. In this application, for simplicity, we neglect $B_{, \beta}^{\alpha \gamma}$ in ([.J5). This is equivalent to neglect of the curvature term $q_{, \alpha \beta}^{1}$. Details of the method and numerical calculation can be found in reference [U]]].

We start the calculation from the two well-separated ${ }^{16} \mathrm{O}$ nuclei, to determine a series of solutions $\left\{\left|\phi\left(q^{1}\right)\right\rangle\right\}$ which simultaneously satisfy (2.6) and ([2.14). After determining the decoupled path, we calculate $\bar{V}\left(q^{1}\right)$ and $\bar{B}^{11}\left(q^{1}\right)$ on that reaction path. In the left panel of figure $\square$, we show the potential as a function of the quadrupole moment. The Coulomb barrier top is at around $Q_{20} \approx 1000$ $\mathrm{fm}^{2}$. Beyond this point toward the collision, the potential decreases until the system reaches the superdeformed local minimum in ${ }^{32} \mathrm{~S}$, at $Q_{20} \approx 400 \mathrm{fm}^{2}$. The superdeformed local minimum is confirmed by the $Q_{20}$-constrained mean-field calculation which is shown by the dashed line in figure 1 (left). The identical superdeformed state is reached by both calculations. The potential landscape turns out to be similar to the one obtained from the $Q_{20}$-constraint calculation. 

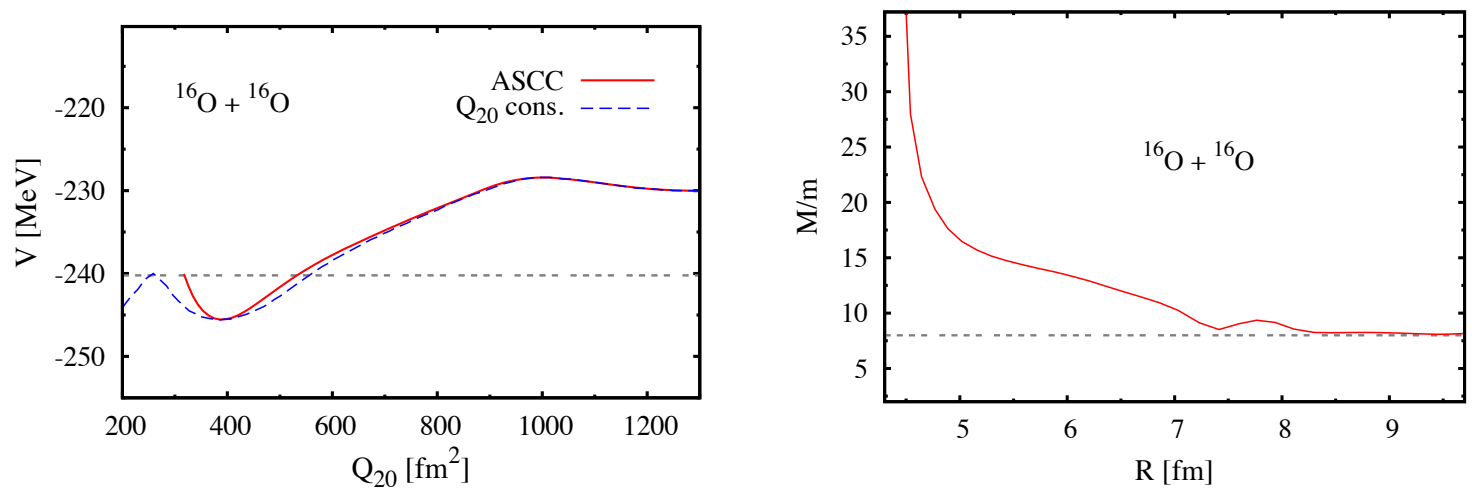

Figure 2: (Left) Potential curve for ${ }^{16} \mathrm{O}+{ }^{16} \mathrm{O}$ scattering as a function of the quadrupole moment $Q_{20}$. The dashed line is the result of the mean-field calculation with $Q_{20}$ as a constraint. The horizontal dotted line indicates twice of the ground-state energy of ${ }^{16} \mathrm{O}$. (Right) Inertial mass for the relative distance $R$ between two ${ }^{16} \mathrm{O}$ nuclei. The dotted line indicates the reduced mass $\mu=8 \mathrm{~m}$.

The inertial mass $M(R)$ for the relative distance $R$ is shown in the right panel of figure $\square$. It reproduces the exact reduced mass $\mu=8 m$, where $m$ is the nucleon mass, at the asymptotic region $(R \rightarrow \infty)$. This means that the relative distance $R$ is indeed proportional to the decoupled collective variable $q^{1}$ in the asymptotic region, $q_{, \alpha}^{1} \| R_{, \alpha}$.

Near the barrier top, the inertial mass $M(R)$ starts to deviate from the reduced mass value $8 m$. As two ${ }^{16} \mathrm{O}$ nuclei approach each other, $M(R)$ increases and becomes close to $20 \mathrm{~m}$ at the superdeformed state. This suggests that the direction of the collective coordinate $q_{, \alpha}^{1}$ becomes significantly different from that of $R$. In fact, beyond the superdeformed minimum, the inertial mass diverges, which indicates $q_{, \alpha}^{1} \perp R_{, \alpha}$. Thus, the relative distance no longer changes and the system cannot reach the ground state of ${ }^{32} \mathrm{~S}$.

In the adiabatic time-dependent HartreeFock theory, the collective path and the inertial mass was obtained for the same system by Reinhard and collaborators [U]]. Their inertial mass behaves similar to ours in the region of $R>6 \mathrm{fm}$, but their result indicates the decrease of the inertial mass at $R<5.5 \mathrm{fm}$. The similar discrepancy was previously found for the $\alpha+\alpha$ system [ए]] .

Adding the centrifugal potential $V_{\text {cen }}^{(l)}(R)=$ $\hbar^{2} l(l+1) /\left(2 \mu R^{2}\right)$ to the obtained collective Hamiltonian, we calculate the transmission coefficient $P_{l}(E)$ for each partial wave $l$, using the generalized WKB formula. The calculated fusion cross section at subbarrier energy is shown in figure B. Since $M(R)$ is larger than the reduced mass $\mu$, the solid line is smaller than the dashed line.

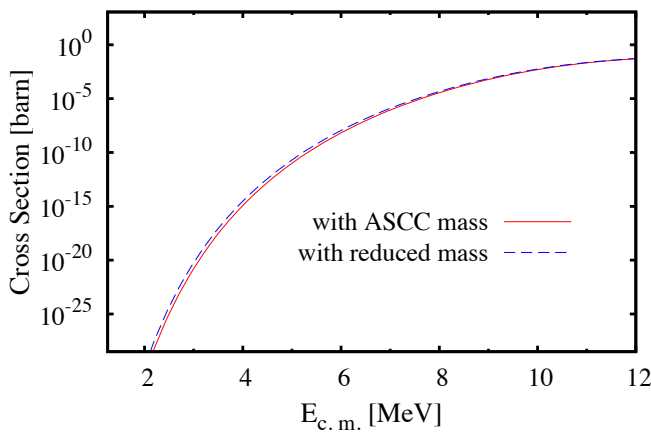

Figure 3: Calculated fusion cross section at subbarrier energies for ${ }^{16} \mathrm{O}+{ }^{16} \mathrm{O}$. The dashed line is obtained by replacing the $R$-dependent inertial mass $M(R)$ with the constant reduced mass $\mu=8 m$. 


\section{Summary}

We presented the adiabatic self-consistent collective coordinate (ASCC) method and its application to nuclear pairing dynamics in $\mathrm{Sn}$ isotopes and low-energy fusion reaction of ${ }^{16} \mathrm{O}+{ }^{16} \mathrm{O}$. The decoupled 1D collective paths were determined and quantized to obtain excitation energies and subbarrier fusion cross section. The collective coordinate for the pairing collective motion cannot be mapped onto the pair gap $\Delta$ because there is no one-to-one correspondence. The collective motion for the fusion reaction asymptotically reduces to the relative motion between two nuclei, while it is very different from the relative motion after two nuclei touch. This leads to a peculiar increase of the inertial mass in the interior region.

\section{Acknowledgments}

This work is supported by JSPS KAKENHI Grant No. 25287065 and by Interdisciplinary Computational Science Program in CCS, University of Tsukuba.

\section{References}

[1] T. Nakatsukasa, K. Matsuyanagi, M. Matsuo, and K. Yabana, Time-dependent density-functional description of nuclear dynamics, Rev. Mod. Phys. 88 (2016) 045004 [arXiv:1606.04717].

[2] M. Matsuo, T. Nakatsukasa, and K. Matsuyanagi, Adiabatic selfconsistent collective coordinate method for large amplitude collective motion in superconducting nuclei, Prog. Theor. Phys. 103 (2000) 959.

[3] P. Ring and P. Schuck, Nuclear many-body problem, Springer-Verlag, New York 1980.

[4] M. Brack, J. Damgaard, A. S. Jensen, H. C. Pauli, V. W. Strutinsky, and C. Y. Wong, Funny hills: The shell-correction approach to nuclear shell effects and its applications to the fission process, Rev.

Mod. Phys. 44 (1972) 320.

[5] K. Kumar and M. Baranger, Nuclear deformations in the pairing-plus-quadrupole model (III), Nucl. Phys. A110 (1968) 529.

[6] T. Nakatsukasa, Density functional approaches to collective phenomena in nuclei: Time-dependent density functional theory for perturbative and non-perturbative nuclear dynamics, Prog. Theor. Exp. Phys. 2012 (2012) 01A207.

[7] G. Do Dang, A. Klein, and N. R. Walet, Self-consistent theory of large-amplitude collective motion: applications to approximate quantization of nonseparable systems and to nuclear physics, Phys. Rep. 335 (2000) 93.

[8] J.-P. Blaizot and G. Ripka, Quantum theory of finite systems, MIT Press, Cambridge, 1986.

[9] P. Bonche and S. Koonin and J. W. Negele, One-dimensional nuclear dynamics in the time-dependent Hartree-Fock approximation, Phys. Rev. C 13 (1976) 1226.

[10] K. Wen and T. Nakatsukasa, Self-consistent collective coordinate for reaction path and inertial mass, Phys. Rev. C 94 (2016) 054618.

[11] P.-G. Reinhard, J. Friedrich, K. Goeke, F. Grümmer, and D. H. E. Gross, Dynamics of the ${ }^{16} \mathrm{O}+{ }^{16} \mathrm{O} \rightarrow{ }^{32} \mathrm{~S}$ fusion process Phys. Rev. C 30 (1984) 878. 